Article

\title{
Fused Heterocyclic Systems with an s-Triazine Ring. 34. Development of a Practical Approach for the Synthesis of 5-Aza-isoguanines
}

\author{
Ahmad Junaid ${ }^{1}$, Felicia Phei Lin Lim ${ }^{1}{ }^{1}$, Yvonne Peijun Zhou ${ }^{2}$, Wai Keung Chui ${ }^{2}$ and \\ Anton V. Dolzhenko 1,3,* \\ 1 School of Pharmacy, Monash University Malaysia, Jalan Lagoon Selatan, Bandar Sunway, \\ Selangor Darul Ehsan 47500, Malaysia; ahmad.junaid@monash.edu (A.J.); felicia.lim@monash.edu (F.P.L.L.) \\ 2 Department of Pharmacy, Faculty of Science, National University of Singapore, 18 Science Drive 4, \\ Singapore 117543, Singapore; marz_vonne@yahoo.com.sg (Y.P.Z.); phacwk@nus.edu.sg (W.K.C.) \\ 3 School of Pharmacy and Biomedical Sciences, Curtin Health Innovation Research Institute, \\ Faculty of Health Sciences, Curtin University, GPO Box U1987, Perth, WA 6845, Australia \\ * Correspondence: anton.dolzhenko@monash.edu; Tel.: +60-3-5514-5867
}

Academic Editors: Panayiotis A. Koutentis and Andreas S. Kalogirou

Received: 12 March 2019; Accepted: 10 April 2019; Published: 12 April 2019

\begin{abstract}
Purine isosteres present excellent opportunities in drug design and development. Using isosteres of natural purines as scaffolds for the construction of new therapeutic agents has been a valid strategy of medicinal chemistry. Inspired by the similarity to isoguanine, we attempted to develop a practical method for the preparation of 5-aza-isoguanines. Several synthetic approaches were explored to establish a robust general protocol for the preparation of these compounds. The significant difference in the reactivity of the C-5 and C-7 electrophilic centers of 1,2,4-triazolo[ $[1,5-a][1,3,5]$ triazines (5-azapurines) towards nucleophiles was demonstrated. The most practical and general method for the preparation of 5-aza-isoguanines involved a regioselective reaction of ethoxycarbonyl isothiocyanate with a 5-aminotriazole. The intramolecular ring closure of the resulted product followed by the S-methylation afforded 7-methylthio-2-phenyl-1,2,4-triazolo[1,5-a][1,3,5]triazin-5-one, which could be effectively aminated with various amines. The resulted 5-aza-isoguanines resemble a known purine nucleoside phosphorylase inhibitor and could be interesting for further investigations as potential anticancer agents.
\end{abstract}

Keywords: triazine; triazole; azapurine; purine isostere

\section{Introduction}

Purine isosteres attract significant attention from medicinal chemists due to their potential for the development of new therapeutic agents. These compounds are known to modulate a complex network of processes, which involves regulatory biogenic purines. The purine-like scaffolds incorporating a 1,3,5-triazine ring became privileged in the construction of bioactive molecules [1]. It was found that pyrazolo[1,5-a][1,3,5]triazine (5-aza-9-deazapurine) [2] and 1,2,4-triazolo[1,5-a][1,3,5]triazine (5-azapurine) [3,4] were the most promising skeletons in this group of purine isosteres. We developed efficient methods for the synthesis of adenine and xanthine analogues built on these scaffolds [5-15]. The synthesis of 5-aza-guanines has also been reported [16,17].

Isoguanine has been reported as a skeleton for several biologically important alkaloids [18-20]. Some interesting results were obtained on a compound with the 4-aminopyrazolo[1,5-a][1,3,5]triazin-2-one skeleton (a triazine-based 5-aza-9-deaza-isostere of isoguanine) [21]. This compound was an efficient inhibitor of purine nucleoside phosphorylase, 
which is a valid target for anticancer and antiparasitic therapy [22-25]. This brought our attention to a similar purine-like heterocyclic system, i.e., 5-aza-isoguanine. Herein, we report our attempts to develop an efficient synthesis of 5-aza-isoguanines bearing various substituents on the amino group.

\section{Results and Discussion}

Earlier we developed convenient syntheses of amino-substituted pyrazolo[1,5- $a][1,3,5]$ triazines and 1,2,4-triazolo[1,5-a][1,3,5]triazines exploiting good reactivity of highly electrophilic 1,3,5-triazine ring substituted with the trichloromethyl group [26,27]. The key step in this synthetic approach was the ring closure reaction of azolylguanidine 1 using trichloroacetonitrile (Scheme 1). The subsequent nucleophilic substitution of the introduced trichloromethyl leaving group of 2 with variety of amines resulted in the formation of the corresponding pyrazolo[ $[1,5-a][1,3,5]$ triazines and 1,2,4-triazolo[1,5-a][1,3,5]triazines 3 .

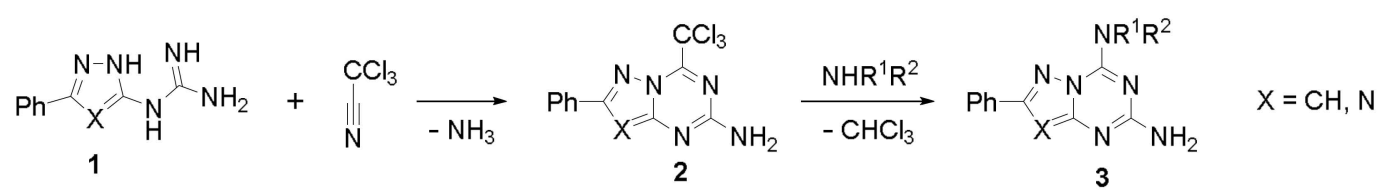

Scheme 1. Synthesis of diamino-substituted azolo[1,5-a][1,3,5]triazines 3.

With our interest leaning towards the synthesis of 5-aza-isoguanines, which are 5-oxo-analogues of compounds 3 , an initial attempt to prepare 1,2,4-triazolo[1,5-a][1,3,5]triazine 5 bearing reactive trichloromethyl group was based on the previously developed reaction using triazolylurea 4 in place of guanidine 1 (Scheme 2). However, despite a number of attempts to achieve the triazine ring closure using trichloroacetonitrile under various reaction conditions, we were able to isolate starting urea 4 only.

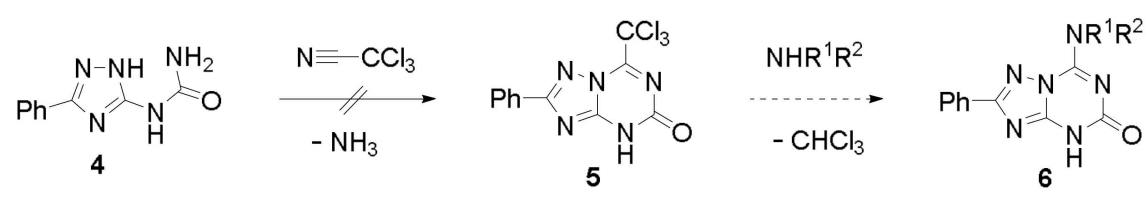

Scheme 2. An attempt to prepare 5-aza-isoguanines via trichloromethyl-substituted intermediate 5.

To further explore the trichloromethyl chemistry in the synthesis of 7-amino-substituted 1,2,4-triazolo[1,5-a][1,3,5]triazin-5-ones 6, we subjected 1-guanyl-1,2,4-triazole 7, prepared as reported previously [28], to the reaction with trichloroacetonitrile (Scheme 3). Similar to triazolylguanidine $1(X=N)$, the reaction pathway of 7 was solvent dependent. In ethanol, diamine 8, identical to that reported in reference [29], was formed, whereas 1,2,4-triazolo[1,5-a][1,3,5]triazine 9 was isolated exclusively when the reaction was carried out in toluene.

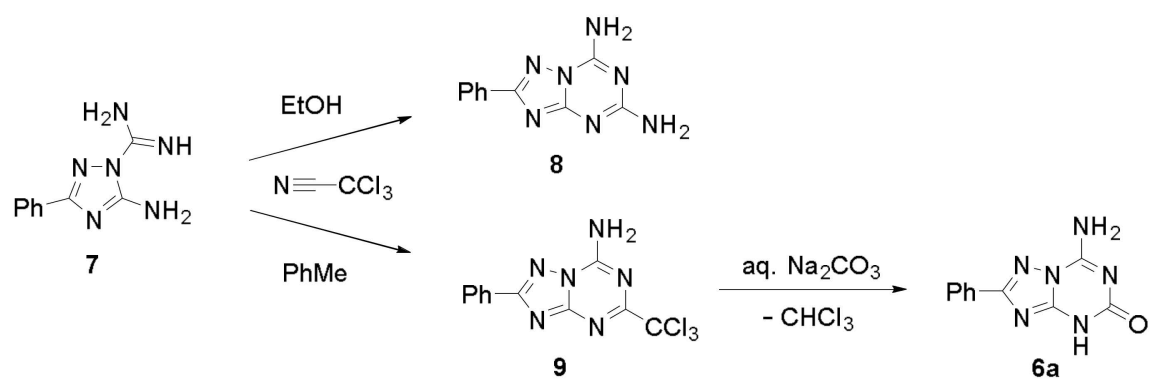

Scheme 3. Synthesis of 5-aza-isoguanine 6a. 
In the ${ }^{1} \mathrm{H}-\mathrm{NMR}$ spectrum of $\mathbf{9}$, two protons of the amino group gave independent signals appearing at rather low field: 9.22 and $9.65 \mathrm{ppm}$. These observations can be attributed to the prominent delocalisation of an electron pair on the amino group nitrogen directly attached to the highly electron-deficient 1,3,5-triazine ring. The electron-withdrawing trichloromethyl group further decreased electron density on the triazine ring, enhancing the partial double bond character of the $\mathrm{C}-\mathrm{NH}_{2}$ bond and restricting the rotation around it. The activation energy $\left(\Delta \mathrm{G}^{\ddagger}\right)$ for the hindered rotation around this bond was estimated using dynamic ${ }^{1} \mathrm{H}-\mathrm{NMR}$ spectroscopy and was equal $68.8 \mathrm{~kJ}$ at the coalescence temperature $343 \mathrm{~K}$.

The trichloromethyl group of $\mathbf{9}$ was hydrolytically removed using aqueous solution of sodium carbonate to afford $\mathbf{6 a}$. The substitution of trichloromethyl group of $\mathbf{9}$ with nucleophiles (particularly amines) was more problematic in comparison to the aminolysis of the isomeric structure 2 . No reaction was observed when similar reaction conditions were applied. Moreover, unexpected results were obtained after prolonged heating with excess of amine depending on the reaction conditions and amine structure (Scheme 4). Replacement of the amino group in position 7 of the 1,2,4-triazolo[1,5- $a][1,3,5]$ triazine ring was observed instead of the proposed substitution of the trichloromethyl group of 9 (e.g., in the synthesis of 10) or both the processes took place together (e.g., in the preparation of 11). It was demonstrated earlier that compounds with identical leaving groups in positions 5 and 7 of the 1,2,4-triazolo[1,5-a][1,3,5]triazine ring were suitable for the sequential nucleophilic substitution with amines: first in position 7, then in position 5 [30]. This strategy was successfully applied for the synthesis of bioactive 1,2,4-triazolo[1,5-a][1,3,5]triazines [31,32]. However, to the best of our knowledge, no examples on transaminations at the position 7 of 1,2,4-triazolo[1,5-a][1,3,5]triazines have been reported. For similar pyrazolo[1,5-a][1,3,5]triazines, the replacement of a N-methylanilino substituent in the corresponding position with other amines was effectively employed in drug-discovery programs to prepare a variety of compounds with a diverse substitution pattern [33-40].

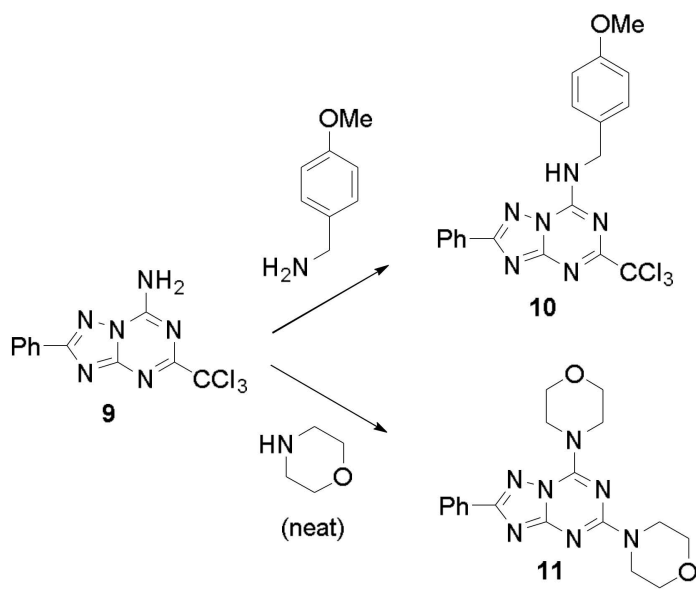

Scheme 4. Reaction of 9 with 4-methoxybenzylamine and morpholine.

The hydrolysis of 7-amino substituted $\mathbf{1 0}$ can be applied to generate 6k. Nevertheless, difficulties with controlling reaction outcome in the synthesis of analogues of $\mathbf{1 0}$ and a long reaction time led us to design an intermediate with a leaving group more accessible for the nucleophilic substitution by amines than 9. This strategy was realized by the preparation of 1,2,4-triazolo[1,5-a][1,3,5] triazine (15) (Scheme 5). Aminotriazole 12 reacted with carbon disulfide in DMF in the presence of potassium hydroxide followed by the selective S-methylation affording 13. Treatment of $\mathbf{1 3}$ with trichloroacetonitrile resulted in the formation of 15. Trichloroacetonitrile provided a $\mathrm{C}-\mathrm{N}$ fragment for the construction of the triazine ring. We propose that the reaction involved an initial addition to the triple bond of trichloroacetonitrile followed by the intramolecular ring closure of intermediate 14 via nucleophilic substitution at the methyl dithiocarboxylate moiety. Surprisingly, hydrogen sulfide instead of methylthiol played the role 
of a leaving group in this reaction. The methylthio group of $\mathbf{1 5}$ can be readily replaced by stoichiometric quantity of amines, e.g., 4-methoxybenzylamine, providing 10. However, a long reaction time at the hydrolysis step arising from the low aqueous solubility of $\mathbf{1 0}$ and the necessity of chromatographic purification limited the practicality of this approach.

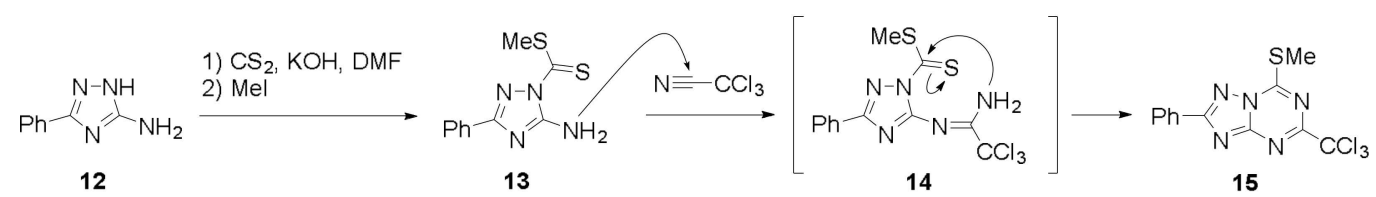

Scheme 5. Synthesis of 5-trichloromethyl-7-methylthio-2-phenyl-1,2,4-triazolo[1,5-a][1,3,5]triazine (15).

Considering the smooth replacement of the methylthio group at position 7 of the 1,2,4-triazolo[1,5-a][1,3,5]triazine with amines, we implemented an alternative synthetic approach for the preparation of 6 . The key intermediate in this protocol was 16, prepared from 5-amino-3-phenyl-1,2,4-triazole (12) (Scheme 6). The selective addition of 12 to ethoxycarbonyl isothiocyanate was challenging due to the presence of several competing nucleophilic centers on 12. It was found that regioselective addition occurs only at the endocyclic N-1 atom of $\mathbf{1 6}$ when the reaction was carried out under kinetic control in cold acetone for not more than 15 min. Extension of the reaction time or increasing temperature led to the formation of more thermodynamically stable $N$-carbethoxy- $N^{\prime}$-(1,2,4-triazol-5-yl)thiourea, which existed in solution as a mixture of two tautomers 17 and $\mathbf{1 7}^{\prime}\left(K_{\mathrm{T}}=0.74\right)$ crystalizing in the form of the predominant tautomer $\mathbf{1 7}$ (Figure 1) [41].

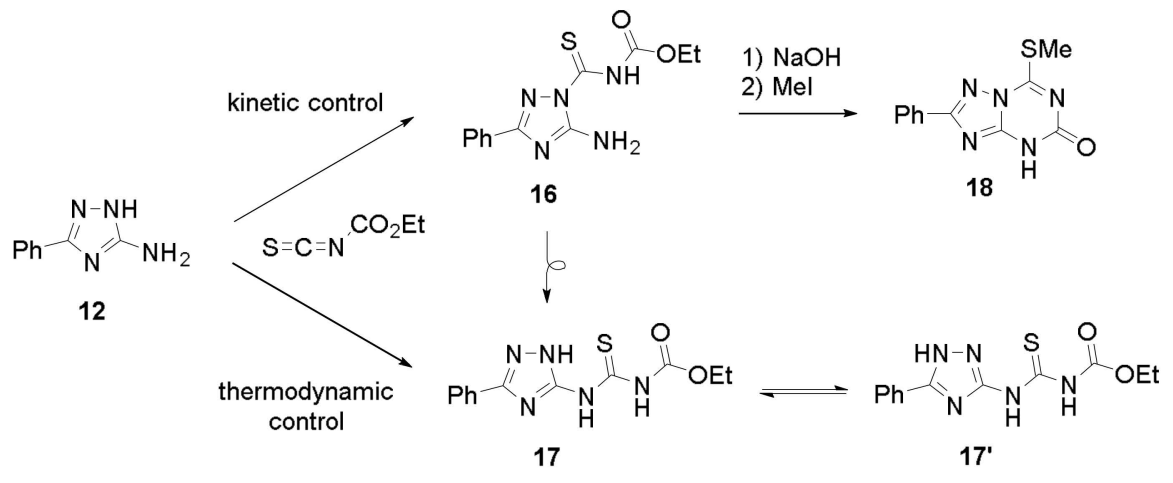

Scheme 6. Synthesis of 7-methylthio-2-phenyl-1,2,4-triazolo[1,5-a][1,3,5]triazin-5-one (18).

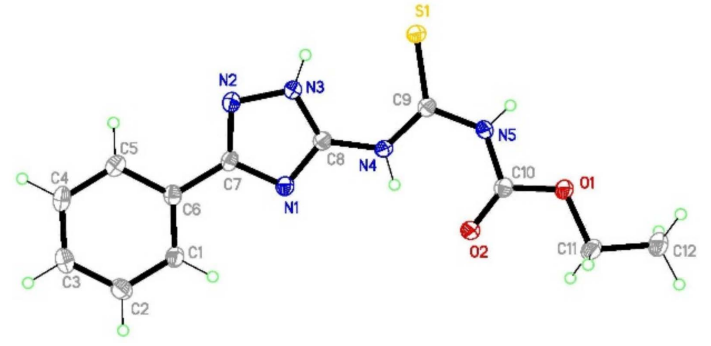

Figure 1. X-ray structure of 17.

In solutions, 16 readily underwent rearrangement to 17 even at room temperature. Therefore, instant preparation of sufficiently pure $\mathbf{1 6}$ was critical, as changes in the product structure during purification were unavoidable. The alkali-induced cyclocondensation of 16 followed by S-methylation afforded 7-methylthio-2-phenyl-1,2,4-triazolo[1,5-a][1,3,5]triazin-5-one (18).

The nucleophilic replacement of the methylthio group of $\mathbf{1 8}$ proceeded smoothly, with a variety of amines affording 6 with reasonable yields and high purity (Scheme 7). The reaction remained 
chemoselective even when aqueous solutions of fairly basic amines (synthesis of $\mathbf{6 a}-\mathbf{c}$ ) were used. The structure of the prepared compounds $\mathbf{6}$ was confirmed by NMR spectroscopy, clearly showing that the signal of methylthio group of $\mathbf{1 8}$ was replaced by signals of the corresponding amines in spectra of $\mathbf{6}$. The X-ray data for a representative example 61 also confirmed the proposed structure (Figure 2) [42].

$$
{ }_{18}
$$

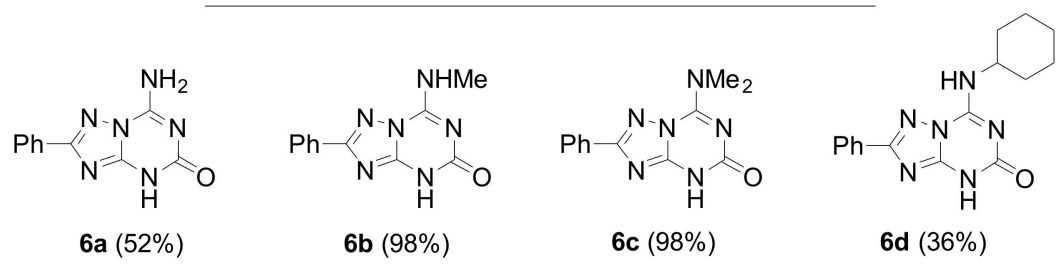

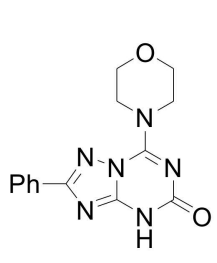

$$
\text { 6h (82\%) }
$$

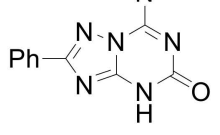

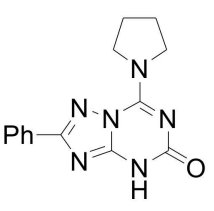

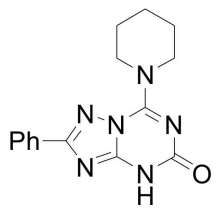

6e $(90 \%)$

$6 f(83 \%)$
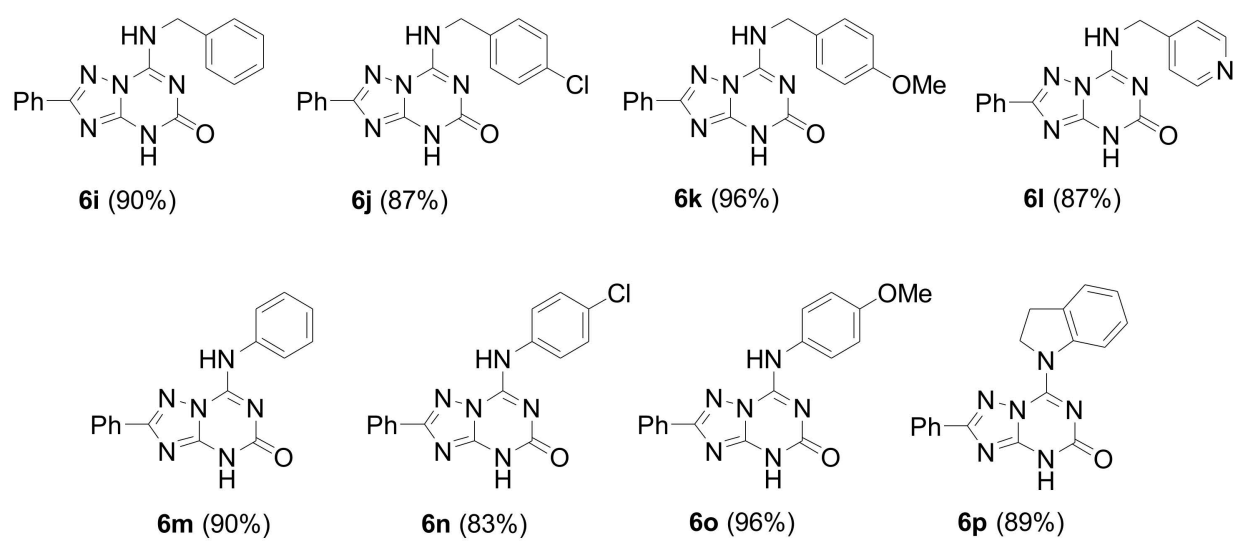

Scheme 7. Synthesis of 5-aza-isoguanines 6.

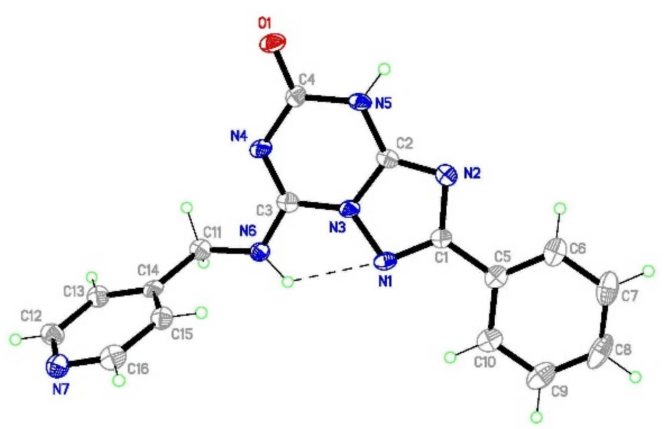

Figure 2. X-ray structure of 61.

The synthetic route described in Schemes 6 and 7 was not placed on the top priority at the initial design of the synthetic routes due to the relatively low yield of the intermediate $\mathbf{1 6}$ and the uncertainty involved in the preparation of a pure regioisomer $\mathbf{1 6}$ as a result of the addition reaction between 
ethoxycarbonyl isothiocyanate to 5-amino-3-phenyl-1,2,4-triazole (12). However, this synthetic route unexpectedly became the most practical and versatile method that could be applied to the synthesis of a library of 7-amino substituted 2-phenyl-1,2,4-triazolo[1,5-a][1,3,5]triazin-5-ones (6a-p).

\section{Conclusions}

In conclusion, several synthetic approaches to the synthesis of 5-aza-isoguanines 6 were explored to develop a practical method for their preparation. The significant difference in the reactivity of the C-5 and C-7 electrophilic centers of 1,2,4-triazolo[1,5-a][1,3,5]triazines towards nucleophiles was demonstrated. The most practical and general method for the preparation of 5-aza-isoguanines 6 involved a regioselective addition of 5-amino-3-phenyl-1,2,4-triazole (12) to ethoxycarbonyl isothiocyanate followed by an intramolecular ring closure and S-methylation to afford 18, which could be effectively aminated with various amines. The prepared compound 6 resemble a known purine nucleoside phosphorylase inhibitor and could be interesting for further investigations as potential anticancer agents.

\section{Materials and Methods}

\subsection{General}

The melting points (uncorrected) were determined via the use of the Gallenkamp melting point apparatus. The ${ }^{1} \mathrm{H}$ NMR and ${ }^{13} \mathrm{C}$ NMR spectra were recorded on a Bruker DPX-300 spectrometer (Fällanden, Switzerland), using DMSO- $d_{6}$ as a solvent and TMS as the internal reference. All the reactions were monitored with the use of the analytical TLC carried out on aluminum plates coated on Silica gel $60 \mathrm{~F}_{254}$ (Merck, Darmstadt, Germany) with detection by UV light.

\subsection{Synthesis}

\subsubsection{Synthesis of 2-Phenyl-1,2,4-triazolo[1,5-a][1,3,5]triazin-5,7-diamine 8}

1-Guanyl-3-phenyl-1,2,4-triazol-5-amine $(7,0.3 \mathrm{~g}, 1.5 \mathrm{mmol})$ and trichloroacetonitrile $(0.45 \mathrm{~mL}$, $4.5 \mathrm{mmol})$ were heated in $\mathrm{EtOH}(10 \mathrm{~mL})$ under reflux for $6 \mathrm{~h}$. After cooling, the precipitated colorless product was filtered and washed with cold $\mathrm{EtOH}$ to give the product, which was found to be identical to compound 8 prepared earlier via an alternative method [29]. Yield $210 \mathrm{mg}, 62 \%$.

\subsubsection{Synthesis of 7-Amino-2-phenyl-5-trichloromethyl-1,2,4-triazolo[1,5-a][1,3,5]triazine 9}

1-Guanyl-3-phenyl-1,2,4-triazol-5-amine (7, $0.3 \mathrm{~g}, 1.5 \mathrm{mmol})$ and trichloroacetonitrile $(0.45 \mathrm{~mL}$, $4.5 \mathrm{mmol})$ were refluxed in toluene $(10 \mathrm{~mL})$ for $6 \mathrm{~h}$. After cooling, the colorless product was filtered and recrystalized from EtOH. Yield $310 \mathrm{mg}, 63 \%$; mp $>300{ }^{\circ} \mathrm{C}(\mathrm{EtOH}) .{ }^{1} \mathrm{H}-\mathrm{NMR}\left(300 \mathrm{MHz}, \mathrm{DMSO}-d_{6}\right)$ : 8 7.53-7.65 (3H, m, H-3' ${ }^{\prime} \mathrm{H}-4^{\prime}$ and H-5' $), 8.19-8.28\left(2 \mathrm{H}, \mathrm{m}, \mathrm{H}-2^{\prime}\right.$ and $\left.\mathrm{H}-6^{\prime}\right), 9.22(1 \mathrm{H}, \mathrm{s}, \mathrm{NH}), 9.65(1 \mathrm{H}$, s, NH). ${ }^{13}$ C-NMR (75 MHz, DMSO- $\left.d_{6}\right): \delta 96.2,126.9$ (2C), 128.9 (2C), 129.7, 130.9, 151.6, 157.2, 164.1, 164.6. Combustion elemental analysis calculated for $\mathrm{C}_{11} \mathrm{H}_{7} \mathrm{Cl}_{3} \mathrm{~N}_{6}$ : $\mathrm{C}, 40.09 ; \mathrm{H}, 2.14 ; \mathrm{N}, 25.50$. Found: C, 39.96; H, 2.35; N, 25.32 .

\subsubsection{Synthesis of 7-Amino-2-phenyl-1,2,4-triazolo[1,5-a][1,3,5]triazin-5-ones $\mathbf{6 a}$ from 9}

To $10 \%$ aqueous $\mathrm{Na}_{2} \mathrm{CO}_{3}(10 \mathrm{~mL})$, 7-amino-2-phenyl-5-trichloromethyl-1,2,4-triazolo[1,5-a][1,3,5] triazine $(9,0.33 \mathrm{~g}, 1 \mathrm{mmol})$ was added, and the mixture was heated under reflux for $72 \mathrm{~h}$. The reaction mixture was allowed to cool and $\mathrm{HCl}(1 \mathrm{~N})$ was added dropwise to $\mathrm{pH} 4$. The precipitate formed was filtered and washed with water to give colorless crystalline powder. Yield $36 \mathrm{mg}, 16 \% ; \mathrm{mp}>300^{\circ} \mathrm{C}$ $\left(\mathrm{MeOCH}_{2} \mathrm{CH}_{2} \mathrm{OH}\right) .{ }^{1} \mathrm{H}-\mathrm{NMR}\left(300 \mathrm{MHz}\right.$, DMSO- $\left.d_{6}\right): \delta$ 7.48-7.58 (3H, m, H-3', H-4' and $\left.\mathrm{H}-5^{\prime}\right), 8.01-8.11$ $\left(2 \mathrm{H}, \mathrm{m}, \mathrm{H}-2^{\prime}\right.$ and $\left.\mathrm{H}-6^{\prime}\right), 8.26(1 \mathrm{H}, \mathrm{s}, \mathrm{NH}), 8.64(1 \mathrm{H}$, brs, $\mathrm{NH}), 12.17(1 \mathrm{H}, \mathrm{brs}, \mathrm{N}(4) \mathrm{H}) .{ }^{13} \mathrm{C}-\mathrm{NMR}(75 \mathrm{MHz}$, DMSO-d $\left._{6}\right): \delta 126.5(2 \mathrm{C}), 128.8(2 \mathrm{C}), 129.6,130.4,149.8,153.1,153.6(2 \mathrm{C}), 161.4$. Combustion elemental analysis for $\mathrm{C}_{10} \mathrm{H}_{8} \mathrm{~N}_{6} \mathrm{O}: \mathrm{C}, 52.63 ; \mathrm{H}, 3.53 ; \mathrm{N}, 36.83$. Found: $\mathrm{C}, 52.42 ; \mathrm{H}, 3.71 ; \mathrm{N}, 36.66$. 
4.2.4. Synthesis of $N$-(4-Methoxybenzyl)-2-phenyl-5-trichloromethyl-1,2,4-triazolo[1,5-a][1,3,5] triazin-7-amine 10

To the suspension of 9 ( $0.33 \mathrm{~g}, 1 \mathrm{mmol})$ in EtOH $(5 \mathrm{~mL})$, 4-methoxybenzylamine ( $0.36 \mathrm{~mL}, 3 \mathrm{mmol})$ was added and the mixture was heated under reflux for $24 \mathrm{~h}$. After cooling, the resulting precipitate was filtered, washed with cold EtOH, dried and recrystallized from EtOH. Yield $212 \mathrm{mg}$, 47\%; mp 174-175 ${ }^{\circ} \mathrm{C}(\mathrm{EtOH}) .{ }^{1} \mathrm{H}-\mathrm{NMR}\left(300 \mathrm{MHz}, \mathrm{DMSO}-d_{6}\right): \delta 3.73(3 \mathrm{H}, \mathrm{s}, \mathrm{OMe}), 4.73\left(2 \mathrm{H}, \mathrm{d}, J=5.8 \mathrm{~Hz}, \mathrm{CH}_{2}\right)$, $6.91\left(2 \mathrm{H}, \mathrm{d}, J=8.7 \mathrm{~Hz}, \mathrm{C}-3^{\prime \prime}\right.$ and C-5" $), 7.47\left(2 \mathrm{H}, \mathrm{d}, J=8.7 \mathrm{~Hz}, \mathrm{C}-2^{\prime \prime}\right.$ and C-6" $\left.{ }^{\prime \prime}\right), 7.52-7.61\left(3 \mathrm{H}, \mathrm{m}, \mathrm{H}-3^{\prime}\right.$, H-4 $4^{\prime}$ and $\left.\mathrm{H}-5^{\prime}\right), 8.16-8.28\left(2 \mathrm{H}, \mathrm{m}, \mathrm{H}-2^{\prime}\right.$ and $\left.\mathrm{H}-6^{\prime}\right), 10.20(1 \mathrm{H}, \mathrm{t}, J=5.8 \mathrm{~Hz}, \mathrm{NH}) .{ }^{13} \mathrm{C}-\mathrm{NMR}(75 \mathrm{MHz}$, DMSO- $\left.\left.d_{6}\right): \delta 43.7,55.0,96.1,113.7\left(2 C^{\prime}\right), 126.9(2 C), 128.9(2 C), 129.3,129.7,129.8(2 C), 130.9\right), 149.6$, 157.1, 158.7, 163.9, 164.5. Combustion elemental analysis calculated for $\mathrm{C}_{19} \mathrm{H}_{15} \mathrm{Cl}_{3} \mathrm{~N}_{6} \mathrm{O}: \mathrm{C}, 50.74 ; \mathrm{H}, 3.36$; $\mathrm{N}, 18.69$. Found: C, 50.55; H, 3.52; N, 18.42.

\subsubsection{Synthesis of 5,7-Bis(morpholino)-2-phenyl-1,2,4-triazolo[1,5-a][1,3,5]triazine $\mathbf{1 1}$}

To $5 \mathrm{~mL}$ of morpholine, $9(0.33 \mathrm{~g}, 1 \mathrm{mmol})$ was added, and the mixture was heated under reflux for $5 \mathrm{~h}$. After cooling, the resulting precipitate was filtered, washed with cold $\mathrm{EtOH}$, dried and recrystallized from EtOH. Yield $180 \mathrm{mg}$, 49\%; mp 278-279 ${ }^{\circ} \mathrm{C}(\mathrm{EtOH}) .{ }^{1} \mathrm{H}-\mathrm{NMR}\left(300 \mathrm{MHz}, \mathrm{DMSO}-d_{6}\right)$ : 8 3.61-3.85 (12H, m), $4.27\left(4 \mathrm{H}\right.$, br. s, $\left.\mathrm{W}_{1 / 2}=24.9 \mathrm{~Hz}\right), 7.46-7.55\left(3 \mathrm{H}, \mathrm{m}, \mathrm{H}-3^{\prime}, \mathrm{H}-4^{\prime}\right.$ and $\left.\mathrm{H}-5^{\prime}\right), 8.05-8.15$ $\left(2 \mathrm{H}, \mathrm{m}, \mathrm{H}-2^{\prime}\right.$ and H-6 $\left.{ }^{\prime}\right) .{ }^{13} \mathrm{C}-\mathrm{NMR}\left(75 \mathrm{MHz}\right.$, DMSO- $\left.d_{6}\right): \delta 44.0$ (br. s), 46.7, 65.7, 65.8, $126.6(2 \mathrm{C}), 128.6$ (2C), $130.2(2 \mathrm{C}), 148.5,158.8,161.1,162.1$. Combustion elemental analysis calculated for $\mathrm{C}_{18} \mathrm{H}_{21} \mathrm{~N}_{7} \mathrm{O}_{2}$ : C, 58.84; H, 5.76; N, 26.69. Found: C, 58.61; H, 6.02; N, 26.41.

\subsubsection{Synthesis of Methyl 5-Amino-3-phenyl-1,2,4-triazoyl-1-dithiocarbonate $\mathbf{1 3}$}

3-Phenyl-1,2,4-triazol-5-amine (12,3.20 g, $20 \mathrm{mmol})$ was dissolved in cold DMF (8 mL). Carbon disulphide $(1.2 \mathrm{~mL}, 20 \mathrm{mmol})$ was added drop-wise, followed by $10 \mathrm{~N} \mathrm{KOH}$ solution $(2 \mathrm{~mL}, 20 \mathrm{mmol})$. The reaction mixture was stirred for $1 \mathrm{~h}$ on an ice-bath. Iodomethane $(1.25 \mathrm{~mL}, 20 \mathrm{mmol})$ was added, and stirring was continued for $10 \mathrm{~min}$. Subsequently, the ice-bath was removed and the reaction mixture was stirred for $2 \mathrm{~h}$. Cold water $(15 \mathrm{~mL})$ was added to the mixture, the yellow precipitate was filtered, washed with water, dried and recrystalized from EtOH. Yield $80 \mathrm{mg}, 32 \%$; mp 207-209 ${ }^{\circ} \mathrm{C}$ (EtOH). ${ }^{1} \mathrm{H}-\mathrm{NMR}\left(300 \mathrm{MHz}, \mathrm{DMSO}-d_{6}\right): \delta 2.63(3 \mathrm{H}, \mathrm{s}, \mathrm{SMe}), 7.47-7.57\left(3 \mathrm{H}, \mathrm{m}, \mathrm{H}-3^{\prime}, \mathrm{H}-4^{\prime}\right.$ and H-5'), $8.03\left(2 \mathrm{H}, \mathrm{dd}, J=6.8,3.0 \mathrm{~Hz}, \mathrm{H}-2^{\prime}\right.$ and $\left.\mathrm{H}-6^{\prime}\right), 8.64\left(2 \mathrm{H}, \mathrm{s}, \mathrm{NH}_{2}\right) .{ }^{13} \mathrm{C}-\mathrm{NMR}\left(75 \mathrm{MHz}, \mathrm{DMSO}-d_{6}\right): \delta 18.8$, 126.6 (2C), 128.8 (2C), 129.1, 130.6, 157.5, 158.8, 199.5. Combustion elemental analysis calculated for $\mathrm{C}_{10} \mathrm{H}_{10} \mathrm{~N}_{4} \mathrm{~S}_{2}: \mathrm{C}, 47.98 ; \mathrm{H}, 4.03 ; \mathrm{N}, 22.38$. Found: $\mathrm{C}, 48.06 ; \mathrm{H}, 4.12 ; \mathrm{N}, 22.14$.

\subsubsection{Synthesis of 7-Methylthio-2-phenyl-5-trichloromethyl-1,2,4-triazolo[1,5- $a][1,3,5]$ triazine 15}

Methyl 5-amino-3-phenyl-1,2,4-triazoyl-1-dithiocarbonate $(\mathbf{1 3}, 0.75 \mathrm{~g}, 3 \mathrm{mmol})$ and trichloroacetonitrile $(0.75 \mathrm{~mL}, 7.5 \mathrm{mmol})$ were heated under reflux in toluene $(10 \mathrm{~mL})$ for $12 \mathrm{~h}$. Solvent was evaporated under reduced pressure, the residue was triturated with diethyl ether and solid was collected by filtration. Yield $245 \mathrm{mg}, 68 \% .{ }^{1} \mathrm{H}-\mathrm{NMR}\left(300 \mathrm{MHz}, \mathrm{DMSO}-d_{6}\right): \delta 2.85(3 \mathrm{H}, \mathrm{s}, \mathrm{SMe})$, 7.56-7.68 (3H, m, H-3', $\mathrm{H}-4^{\prime}$ and $\left.\mathrm{H}-5^{\prime}\right), 8.26\left(2 \mathrm{H}, \mathrm{dd}, J=6.6,2.8 \mathrm{~Hz}, \mathrm{H}-2^{\prime}\right.$ and $\left.\mathrm{H}-6^{\prime}\right) .{ }^{13} \mathrm{C}-\mathrm{NMR}(75 \mathrm{MHz}$, DMSO-d $_{6}$ ): $\delta 13.1,95.2,127.2(2 \mathrm{C}), 129.0,129.1$ (2C), 131.6, 155.2, 161.2, 164.4, 166.0. Combustion elemental analysis calculated for $\mathrm{C}_{12} \mathrm{H}_{8} \mathrm{Cl}_{3} \mathrm{~N}_{5} \mathrm{~S}$ : C, 39.97; H, 2.24; N, 19.42. Found: $\mathrm{C}, 40.12 ; \mathrm{H}, 2.33$; N, 19.11.

\subsubsection{Reactions of 3-Phenyl-1,2,4-triazol-5-amine (12) with Ethoxycarbonyl Isothiocyanate}

Synthesis of 5-amino-1-carbethoxythiocarbamoyl-1,2,4-triazole (16). To a solution of acetone (30 mL), 3-phenyl-1,2,4-triazol-5-amine (12,3.20 g, $20 \mathrm{mmol})$ and ethoxycarbonyl isothiocyanate $(2.62 \mathrm{~mL}, 20 \mathrm{mmol})$ were added. The reaction mixture was stirred on an ice-bath for $15 \mathrm{~min}$. The yellow precipitate formed was filtered immediately. Yield $2.16 \mathrm{~g}, 37 \%$; mp $207-209{ }^{\circ} \mathrm{C}$. ${ }^{1} \mathrm{H}-\mathrm{NMR}(300 \mathrm{MHz}$, DMSO- $\left.d_{6}\right): \delta 2.63(3 \mathrm{H}, \mathrm{s}, \mathrm{SMe}), 7.47-7.57\left(3 \mathrm{H}, \mathrm{m}, \mathrm{H}-3^{\prime}, \mathrm{H}^{-} 4^{\prime}\right.$ and $\left.\mathrm{H}-5^{\prime}\right), 8.03(2 \mathrm{H}, \mathrm{dd}, J=6.8,3.0 \mathrm{~Hz}$, 
H-2' and H-6' $), 8.64\left(2 \mathrm{H}, \mathrm{s}, \mathrm{NH}_{2}\right) .{ }^{13} \mathrm{C}-\mathrm{NMR}\left(75 \mathrm{MHz}^{\prime}\right.$ DMSO-d 6 ): $\delta 18.8,126.6(2 \mathrm{C}), 128.8(2 \mathrm{C}), 129.1$, 130.6, 157.5, 158.8, 199.5. Combustion elemental analysis calculated for $\mathrm{C}_{12} \mathrm{H}_{13} \mathrm{~N}_{5} \mathrm{O}_{2} \mathrm{~S}: \mathrm{C}, 49.47 ; \mathrm{H}, 4.50$; N, 24.04. Found: C, 49.20; H, 4.67; N, 23.88.

Synthesis of $N$-carbethoxy- $N^{\prime}$-(3-phenyl-1H-1,2,4-triazol-5-yl)thiourea (17). To the solution of 3-phenyl-1,2,4-triazol-5-amine (12, $0.48 \mathrm{~g}, 3 \mathrm{mmol})$ in anhydrous DMF (4 mL), ethoxycarbonyl isothiocyanate $(0.43 \mathrm{~mL}, 3.3 \mathrm{mmol})$ was added. After stirring the mixture for $5 \mathrm{~h}$ at room temperature, cold water $(60 \mathrm{~mL})$ was added. The precipitated product was filtered, washed with cold water and recrystalized from EtOH. Yield $0.74 \mathrm{~g}, 85 \%$; mp 180-181 ${ }^{\circ} \mathrm{C}(\mathrm{EtOH}) .{ }^{1} \mathrm{H}-\mathrm{NMR}\left(300 \mathrm{MHz}, \mathrm{DMSO}-\mathrm{d}_{6}\right): \delta$ $1.28\left(3 \mathrm{H}, \mathrm{t}, J=7.1 \mathrm{~Hz}, \mathrm{CH}_{3}\right), 4.25\left(2 \mathrm{H}, \mathrm{q}, J=7.1 \mathrm{~Hz}, \mathrm{CH}_{2}\right), 7.37-7.66\left(3 \mathrm{H}, \mathrm{m}, \mathrm{H}-3^{\prime}, \mathrm{H}-4^{\prime}\right.$ and H-5' $), 8.01$ $\left(2 \mathrm{H}, \mathrm{d}, J=7.2 \mathrm{~Hz}, \mathrm{H}-2^{\prime}\right.$ and H-6 $), 11.56^{*}(12 \mathrm{H}$, brs, 2NH), $11.87(1 \mathrm{H}$, brs, NH), 12.16 (1H, brs, NH), 13.93 $(1 \mathrm{H}$, brs, $\mathrm{N}(1) \mathrm{H}), 14.47(1 \mathrm{H}, \text { brs, } \mathrm{N}(1) \mathrm{H})^{*}$. * - signals of the minor tautomer. Combustion elemental analysis calculated for $\mathrm{C}_{12} \mathrm{H}_{13} \mathrm{~N}_{5} \mathrm{O}_{2} \mathrm{~S}$ : C, 49.47; H, 4.50; N, 24.04. Found: C, 49.33; H, 4.62; N, 23.95.

\subsubsection{Synthesis of 7-Methylthio-2-phenyl-1,2,4-triazolo[1,5-a][1,3,5]triazin-5-one 18}

5-Amino-1-carbethoxythiocarbamoyl-1,2,4-triazole (16, $2.9 \mathrm{~g}, 10 \mathrm{mmol})$ was heated under reflux in aqueous $\mathrm{EtOH}(80 \%, 20 \mathrm{~mL})$ for $20 \mathrm{~min}$ and then cooled. The creamy product was filtered, dissolved in $5 \mathrm{~mL}$ of water and then stirred with iodomethane $(0.62 \mathrm{~mL}, 10 \mathrm{mmol})$ for $30 \mathrm{~min}$ at $10{ }^{\circ} \mathrm{C}$ with a slow warming to room temperature. The colorless precipitate formed was filtered and dissolved in water $(30 \mathrm{~mL})$. A solution of $\mathrm{HCl}(1 \mathrm{~N})$ was then added drop-wise to $\mathrm{pH}$. The colorless precipitate formed was filtered, washed with water and dried. Yield $1.68 \mathrm{~g}, 65 \% ; \mathrm{mp} 275-276{ }^{\circ} \mathrm{C} .{ }^{1} \mathrm{H}-\mathrm{NMR}(300 \mathrm{MHz}$, DMSO-d $\left.d_{6}\right): \delta 2.62(3 \mathrm{H}, \mathrm{s}, \mathrm{SMe}), 7.49-7.59\left(3 \mathrm{H}, \mathrm{m}, \mathrm{H}-3^{\prime}, \mathrm{H}-4^{\prime}\right.$ and H-5'), 8.00-8.11 (2H, m, H-2' and H-6 $\left.{ }^{\prime}\right), 13.07$ (1H, s, N(4)H). ${ }^{13}$ C-NMR (75 MHz, DMSO-d 6 ): $\delta 12.8,126.7$ (2C), 128.9 (2C), 130.0, 130.8, 151.2, 152.1 (2C), 162.2, 162.6. Combustion elemental analysis calculated for $\mathrm{C}_{11} \mathrm{H}_{9} \mathrm{~N}_{5} \mathrm{OS}$ : C, 50.96; H, 3.50; N, 27.01. Found: C, 50.77; H, 3.62; N, 26.90.

\subsubsection{Synthesis of 7-Amino-2-phenyl-1,2,4-triazolo[1,5-a][1,3,5]triazin-5-one 6a from 18}

7-Methylthio-2-phenyl-1,2,4-triazolo[1,5-a][1,3,5]triazin-5-one (18, 0.52 g, $2 \mathrm{mmol})$ was added to a solution of aqueous $\mathrm{NH}_{3}(28 \%, 20 \mathrm{~mL})$, and the mixture was stirred on a water bath at $55{ }^{\circ} \mathrm{C}$ for $18 \mathrm{~h}$. The colorless precipitate formed was filtered and recrystalized from $\mathrm{MeOCH}_{2} \mathrm{CH}_{2} \mathrm{OH}$. Yield $238 \mathrm{mg}$, $52 \%$. Analytical data obtained were identical to those of compound 6 a obtained from the method described under Section 4.2.3.

\subsubsection{Synthesis of 7-Amino-substituted-2-phenyl-1,2,4-triazolo[1,5-a][1,3,5]triazin-5-ones $\mathbf{6 b}$ and $\mathbf{6 c}$}

7-Methylthio-2-phenyl-1,2,4-triazolo[1,5- $a][1,3,5]$ triazin-5-one (18, $0.52 \mathrm{~g}, 2 \mathrm{mmol})$ was added to an aqueous solution of methylamine $(40 \%)$ or dimethylamine $(40 \%)(10 \mathrm{~mL})$ and the mixture was stirred at room temperature for $12 \mathrm{~h}$. Solvent was evaporated under reduced pressure and recrystalized from a suitable solvent.

7-Methylamino-2-phenyl-1,2,4-triazolo[1,5-a][1,3,5]triazine-5-one (6b). Colorless crystalline powder; yield $474 \mathrm{mg}, 98 \%$; mp 298-299 ${ }^{\circ} \mathrm{C}(\mathrm{EtOH}) ;{ }^{1} \mathrm{H}-\mathrm{NMR}\left(300 \mathrm{MHz}, \mathrm{DMSO}-d_{6}\right): \delta 2.94(3 \mathrm{H}, \mathrm{d}, J=3.8 \mathrm{~Hz}, \mathrm{NHMe})$, 7.48-7.58 (3H, m, H-3' ${ }^{\prime} \mathrm{H}-4^{\prime}$ and $\left.\mathrm{H}^{-} 5^{\prime}\right), 8.02-8.11\left(2 \mathrm{H}, \mathrm{m}, \mathrm{H}-2^{\prime}\right.$ and $\left.\mathrm{H}-6^{\prime}\right), 8.71(1 \mathrm{H}, \mathrm{q}, J=3.8 \mathrm{~Hz}, \mathrm{NH})$, $12.18(1 \mathrm{H}, \mathrm{s}, \mathrm{N}(4) \mathrm{H}) .{ }^{13} \mathrm{C}-\mathrm{NMR}\left(75 \mathrm{MHz}, \mathrm{DMSO}-d_{6}\right): \delta 27.3,126.5$ (2C), $128.8(2 \mathrm{C}), 129.6,130.4,148.3$, 152.8, 153.4 (brs), 161.3. Combustion elemental analysis calculated for $\mathrm{C}_{11} \mathrm{H}_{10} \mathrm{~N}_{6} \mathrm{O}: \mathrm{C}, 54.54 ; \mathrm{H}, 4.16 ; \mathrm{N}$, 34.69. Found: C, 54.46; H, 4.21; N, 34.60.

7-Dimethylamino-2-phenyl-1,2,4-triazolo[1,5-a][1,3,5]triazine-5-one (6c). Colorless crystalline powder; yield $500 \mathrm{mg}, 98 \%$; mp > $300{ }^{\circ} \mathrm{C}\left(\mathrm{MeOCH}_{2} \mathrm{CH}_{2} \mathrm{OH}\right) ;{ }^{1} \mathrm{H}-\mathrm{NMR}\left(300 \mathrm{MHz}, \mathrm{DMSO}-d_{6}\right): \delta 3.47(6 \mathrm{H}, \mathrm{brs}$, $\left.\mathrm{NMe}_{2}\right)$, 7.49-7.57 (3H, m, H-3' ${ }^{\prime} \mathrm{H}-4^{\prime}$ and H-5' $), 8.00-8.10\left(2 \mathrm{H}, \mathrm{m}, \mathrm{H}-2^{\prime}\right.$ and H-6' $), 12.24(1 \mathrm{H}, \mathrm{s}, \mathrm{N}(4) \mathrm{H})$. Combustion elemental analysis calculated for $\mathrm{C}_{12} \mathrm{H}_{12} \mathrm{~N}_{6} \mathrm{O}: \mathrm{C}, 56.24 ; \mathrm{H}, 4.72 ; \mathrm{N}, 32.79$. Found: $\mathrm{C}, 56.11$; $\mathrm{H}, 4.89 ; \mathrm{N}, 32.62$. 
4.2.12. Synthesis of 7-Amino-substituted-2-phenyl-1,2,4-triazolo[1,5- $a][1,3,5]$ triazin-5-ones $6 \mathbf{d}-6 \mathbf{p}$.

7-Methylthio-2-phenyl-1,2,4-triazolo[1,5-a][1,3,5]triazin-5-one (18, $0.52 \mathrm{~g}, 2 \mathrm{mmol})$ was added to a solution of appropriate amine $(3 \mathrm{mmol})$ in DMF $(5 \mathrm{~mL})$, and the mixture was heated at $70-80{ }^{\circ} \mathrm{C}$ with stirring for 4-14 h. After cooling, ice cold water $(40 \mathrm{~mL})$ was added and the product was filtered and recrystalized from a suitable solvent.

7-Cyclohexylamino-2-phenyl-1,2,4-triazolo[1,5-a][1,3,5]triazin-5-one (6d). Colorless crystalline powder; yield $245 \mathrm{mg}, 36 \%$; $\mathrm{mp}>300{ }^{\circ} \mathrm{C}(\mathrm{EtOH}) .{ }^{1} \mathrm{H}-\mathrm{NMR}\left(300 \mathrm{MHz}, \mathrm{DMSO}-d_{6}\right): \delta 1.03-1.21\left(1 \mathrm{H}, \mathrm{m}, \mathrm{H}_{\mathrm{c}-\mathrm{Hex}}\right)$, 1.23-1.42 (2H, m, $\left.\mathrm{H}_{\mathrm{c}-\mathrm{Hex}}\right), 1.44-1.69\left(3 \mathrm{H}, \mathrm{m}, \mathrm{H}_{\mathrm{c}-\mathrm{Hex}}\right), 1.71-1.92\left(4 \mathrm{H}, \mathrm{m}, \mathrm{H}_{\mathrm{c}-\mathrm{Hex}}\right), 3.85-4.01\left(1 \mathrm{H}, \mathrm{m}, \mathrm{H}-1^{\prime \prime}\right)$, 7.47-7.59 (3H, m, H-3' , H-4' and $\left.\mathrm{H}^{\prime} 5^{\prime}\right), 8.02-8.16\left(2 \mathrm{H}, \mathrm{m}, \mathrm{H}-2^{\prime}\right.$ and $\left.\mathrm{H}-6^{\prime}\right), 8.56(1 \mathrm{H}, \mathrm{d}, J=8.3 \mathrm{~Hz}, \mathrm{NH})$, $12.17(1 \mathrm{H}, \mathrm{s}, \mathrm{N}(4) \mathrm{H}) .{ }^{13} \mathrm{C}-\mathrm{NMR}\left(75 \mathrm{MHz}, \mathrm{DMSO}-d_{6}\right): \delta 24.85(2 \mathrm{C}), 24.89,31.5(2 \mathrm{C}), 50.0,126.6$ (2C), 128.7 (2C), 129.6, 130.4, 147.1, 152.9, 153.5 (2C), 161.3. Combustion elemental analysis calculated for $\mathrm{C}_{16} \mathrm{H}_{18} \mathrm{~N}_{6} \mathrm{O}: \mathrm{C}, 61.92 ; \mathrm{H}, 5.85 ; \mathrm{N}, 27.08$. Found: $\mathrm{C}, 61.86 ; \mathrm{H}, 6.02 ; \mathrm{N}, 26.89$.

2-Phenyl-7-pyrrolidino-1,2,4-triazolo[1,5-a][1,3,5]triazin-5-one (6e). Colorless crystalline powder; yield $508 \mathrm{mg}, 90 \%$; mp > $300{ }^{\circ} \mathrm{C}(\mathrm{DMSO}) ;{ }^{1} \mathrm{H}-\mathrm{NMR}\left(300 \mathrm{MHz}, \mathrm{DMSO}-d_{6}\right): \delta 1.82-2.08(4 \mathrm{H}, \mathrm{m}$, $\left.\mathrm{C}\left(3^{\prime \prime}\right) \mathrm{H}_{2} \mathrm{C}\left(4^{\prime \prime}\right) \mathrm{H}_{2}\right), 3.64\left(1 \mathrm{H}, \mathrm{t}, J=6.0 \mathrm{~Hz}, \mathrm{C}\left(5^{\prime \prime}\right) \mathrm{H}_{2}\right), 4.25\left(1 \mathrm{H}, \mathrm{t}, J=6.0 \mathrm{~Hz}, \mathrm{C}\left(2^{\prime \prime}\right) \mathrm{H}_{2}\right), 7.48-7.57$ $\left(3 \mathrm{H}, \mathrm{m}, \mathrm{H}-3^{\prime}, \mathrm{H}-4^{\prime}\right.$ and $\left.\mathrm{H}-5^{\prime}\right), 8.00-8.09$ (2H, m, H-2' and H-6'), $12.15(1 \mathrm{H}, \mathrm{s}, \mathrm{N}(4) \mathrm{H})$. Combustion elemental analysis calculated for $\mathrm{C}_{14} \mathrm{H}_{14} \mathrm{~N}_{6} \mathrm{O}: \mathrm{C}, 59.56 ; \mathrm{H}, 5.00 ; \mathrm{N}, 29.77$. Found: C, 59.43; H, 5.24; $\mathrm{N}, 29.51$.

7-Piperidino-2-phenyl-1,2,4-triazolo[1,5-a][1,3,5]triazin-5-one (6f). Colorless crystalline powder; yield $492 \mathrm{mg}, 83 \%$; mp $286{ }^{\circ} \mathrm{C}\left(\mathrm{MeOCH}_{2} \mathrm{CH}_{2} \mathrm{OH}\right) .{ }^{1} \mathrm{H}-\mathrm{NMR}\left(300 \mathrm{MHz}, \mathrm{DMSO}-d_{6}\right): \delta 1.57-1.78(6 \mathrm{H}, \mathrm{m}$, $\mathrm{C}\left(3^{\prime \prime}\right) \mathrm{H}_{2}, \mathrm{C}\left(4^{\prime \prime}\right) \mathrm{H}_{2}, \mathrm{C}\left(5^{\prime \prime}\right) \mathrm{H}_{2}, 3.80-4.50\left(4 \mathrm{H}, \mathrm{m}, \mathrm{C}\left(2^{\prime \prime}\right) \mathrm{H}_{2}, \mathrm{C}\left(6^{\prime \prime}\right) \mathrm{H}_{2}, 7.47-7.58\left(3 \mathrm{H}, \mathrm{m}, \mathrm{H}-3^{\prime}, \mathrm{H}-4^{\prime}\right.\right.$ and H-5'), 7.98-8.10 (2H, m, H-2' and H-6'), $12.29(1 \mathrm{H}, \mathrm{s}, \mathrm{N}(4) \mathrm{H}) .{ }^{13} \mathrm{C}-\mathrm{NMR}\left(75 \mathrm{MHz}, \mathrm{DMSO}-d_{6}\right): \delta 23.7$, $25.5,47.9,126.5$ (2C), 128.8 (2C), 129.3, 130.5, 147.4, 152.8, 154.9 (2C), 160.4. Combustion elemental analysis calculated for $\mathrm{C}_{15} \mathrm{H}_{16} \mathrm{~N}_{6} \mathrm{O}$ : C, 60.80; H, 5.44; N, 28.36. Found: C, 60.68; H, 5.63; N, 28.21.

7-Morpholino-2-phenyl-1,2,4-triazolo[1,5-a][1,3,5]triazin-5-one (6g). Colorless crystalline powder; yield $573 \mathrm{mg}, 96 \%$; mp > $300{ }^{\circ} \mathrm{C}\left(\mathrm{MeOCH}_{2} \mathrm{CH}_{2} \mathrm{OH}\right) ;{ }^{1} \mathrm{H}-\mathrm{NMR}\left(300 \mathrm{MHz}, \mathrm{DMSO}-d_{6}\right): \delta 3.77(4 \mathrm{H}, \mathrm{t}, J=4.5 \mathrm{~Hz}$, $\left.\mathrm{CH}_{2} \mathrm{OCH}_{2}\right), 4.23\left(4 \mathrm{H}\right.$, brs, $\left.\mathrm{CH}_{2} \mathrm{NCH}_{2}\right), 7.47-7.57\left(3 \mathrm{H}, \mathrm{m}, \mathrm{H}-3^{\prime}, \mathrm{H}^{\prime} 4^{\prime}\right.$ and $\left.\mathrm{H}-5^{\prime}\right), 8.00-8.10\left(2 \mathrm{H}, \mathrm{m}, \mathrm{H}-2^{\prime}\right.$ and $\left.\mathrm{H}-66^{\prime}\right), 12.39(1 \mathrm{H}, \mathrm{s}, \mathrm{N}(4) \mathrm{H}) .{ }^{13} \mathrm{C}-\mathrm{NMR}\left(75 \mathrm{MHz}, \mathrm{DMSO}-d_{6}\right): \delta 47.2$ (brs), 65.7, $126.6(2 \mathrm{C}), 128.8(2 \mathrm{C})$, $129.1,130.6,147.7,152.7,154.9(2 \mathrm{C}), 160.5$. Combustion elemental analysis calculated for $\mathrm{C}_{14} \mathrm{H}_{14} \mathrm{~N}_{6} \mathrm{O}_{2}$ : C, 56.37; H, 4.73; N, 28.17. Found: C, 56.22; H, 4.87; N, 28.02.

7-N-methylpiperazino-2-phenyl-1,2,4-triazolo[1,5-a][1,3,5]triazin-5-one (6h). Colorless crystalline powder; yield $510 \mathrm{mg}$, 82\%; mp 266-268 ${ }^{\circ} \mathrm{C}\left(\mathrm{MeOCH}_{2} \mathrm{CH}_{2} \mathrm{OH}\right) ;{ }^{1} \mathrm{H}-\mathrm{NMR}\left(300 \mathrm{MHz}, \mathrm{DMSO}-d_{6}\right): \delta 2.24(3 \mathrm{H}, \mathrm{s}$, $\mathrm{NMe}), 2.44-2.55\left(4 \mathrm{H}, \mathrm{m}, \mathrm{CH}_{2} \mathrm{~N}(\mathrm{Me}) \mathrm{CH}_{2}\right), 4.24\left(4 \mathrm{H}, \mathrm{brs}, \mathrm{CH}_{2} \mathrm{NCH}_{2}\right), 7.47-7.59\left(3 \mathrm{H}, \mathrm{m}, \mathrm{H}-3^{\prime}, \mathrm{H}^{-} 4^{\prime}\right.$ and H-5' $), 7.99-8.10\left(2 \mathrm{H}, \mathrm{m}, \mathrm{H}-2^{\prime}\right.$ and H-6 $\left.{ }^{\prime}\right), 12.11(1 \mathrm{H}, \mathrm{brs}, \mathrm{N}(4) \mathrm{H}) .{ }^{13} \mathrm{C}-\mathrm{NMR}\left(75 \mathrm{MHz}, \mathrm{DMSO}-d_{6}\right): \delta 45.3$, 46.6 (brs), 54.1, 126.5 (2C), 128.8 (2C), 129.2, 130.6, 147.6, 152.8, 154.9 (2C), 160.5. Combustion elemental analysis calculated for $\mathrm{C}_{15} \mathrm{H}_{17} \mathrm{~N}_{7} \mathrm{O}$ : C, 57.87; $\mathrm{H}, 5.50 ; \mathrm{N}, 31.49$. Found: $\mathrm{C}, 57.64 ; \mathrm{H}, 5.62 ; \mathrm{N}, 31.35$.

7-Benzylamino-2-phenyl-1,2,4-triazolo[1,5-a][1,3,5]triazin-5-one (6i). Colorless crystalline powder; yield $572 \mathrm{mg}, 90 \%$; mp > $300{ }^{\circ} \mathrm{C}(\mathrm{DMF}) ;{ }^{1} \mathrm{H}-\mathrm{NMR}\left(300 \mathrm{MHz}, \mathrm{DMSO}-d_{6}\right): \delta 4.63\left(2 \mathrm{H}, \mathrm{d}, J=6.0 \mathrm{~Hz}, \mathrm{CH}_{2}\right), 7.27$ $\left(1 \mathrm{H}, \mathrm{t}, J=7.0 \mathrm{~Hz}, \mathrm{H}-4^{\prime \prime}\right), 7.35\left(2 \mathrm{H}, \mathrm{t}, J=7.3 \mathrm{~Hz}, \mathrm{H}-3^{\prime \prime}\right.$ and $\left.\mathrm{H}-5^{\prime \prime}\right), 7.41\left(2 \mathrm{H}, \mathrm{d}, J=7.9 \mathrm{~Hz}, \mathrm{H}-2^{\prime \prime}\right.$ and $\left.\mathrm{H}-6^{\prime \prime}\right)$, 7.48-7.59 (3H, m, H-3' , H-4' and H-5' ), 8.03-8.13 (2H, m, H-2' and H-6' $), 9.31(1 \mathrm{H}, \mathrm{t}, J=6.0 \mathrm{~Hz}, \mathrm{NH})$, $12.24(1 \mathrm{H}, \mathrm{s}, \mathrm{N}(4) \mathrm{H}) .{ }^{13} \mathrm{C}-\mathrm{NMR}\left(75 \mathrm{MHz}, \mathrm{DMSO}-d_{6}\right): \delta 43.4,126.5(2 \mathrm{C}), 127.0,127.4(2 \mathrm{C}), 128.2(2 \mathrm{C})$, 128.8 (2C), 129.6, 130.5, 138.0, 148.2, 152.9, 153.5 (2C), 161.4. Combustion elemental analysis calculated for $\mathrm{C}_{17} \mathrm{H}_{14} \mathrm{~N}_{6} \mathrm{O}: \mathrm{C}, 64.14 ; \mathrm{H}, 4.43 ; \mathrm{N}, 26.40$. Found: $\mathrm{C}, 63.89 ; \mathrm{H}, 4.64 ; \mathrm{N}, 26.19$. 
7-(4-Chlorobenzylamino)-2-phenyl-1,2,4-triazolo[1,5-a][1,3,5]triazin-5-one (6j). Colorless crystalline powder; yield $614 \mathrm{mg}, 87 \%$; mp > $300{ }^{\circ} \mathrm{C}(\mathrm{DMF}) ;{ }^{1} \mathrm{H}-\mathrm{NMR}\left(300 \mathrm{MHz}, \mathrm{DMSO}-d_{6}\right): \delta 4.61\left(2 \mathrm{H}, \mathrm{d}, J=5.7 \mathrm{~Hz}, \mathrm{CH}_{2}\right)$, $7.40\left(2 \mathrm{H}, \mathrm{d}, J=8.7 \mathrm{~Hz}, \mathrm{H}-3^{\prime \prime}\right.$ and $\left.\mathrm{H}-5^{\prime \prime}\right), 7.44\left(2 \mathrm{H}, \mathrm{d}, J=8.7 \mathrm{~Hz}, \mathrm{H}-2^{\prime \prime}\right.$ and $\left.\mathrm{H}-6^{\prime \prime}\right), 7.48-7.59\left(3 \mathrm{H}, \mathrm{m}, \mathrm{H}-3^{\prime}\right.$, H-4 $4^{\prime}$ and $\left.\mathrm{H}-5^{\prime}\right), 8.03-8.13\left(2 \mathrm{H}, \mathrm{m}, \mathrm{H}-2^{\prime}\right.$ and $\left.\mathrm{H}-6^{\prime}\right), 9.32(1 \mathrm{H}, \mathrm{t}, J=5.7 \mathrm{~Hz}, \mathrm{NH}), 12.25(1 \mathrm{H}, \mathrm{s}, \mathrm{N}(4) \mathrm{H})$. ${ }^{13} \mathrm{C}-\mathrm{NMR}\left(75 \mathrm{MHz}\right.$, DMSO- $\left.d_{6}\right): \delta 42.8,126.5(2 \mathrm{C}), 128.1(2 \mathrm{C}), 128.8(2 \mathrm{C}), 129.3(2 \mathrm{C}), 129.5,130.5,131.6$, 137.0, 148.2, 152.9, $153.4(2 \mathrm{C}), 161.5$. Combustion elemental analysis calculated for $\mathrm{C}_{17} \mathrm{H}_{13} \mathrm{ClN}_{6} \mathrm{O}: \mathrm{C}$, 57.88; H, 3.71; N, 23.82. Found: C, 57.71; H, 4.00; N, 23.69.

7-(4-Methoxybenzylamino)-2-phenyl-1,2,4-triazolo[1,5-a][1,3,5]triazin-5-one (6k). Colorless crystalline powder; yield $670 \mathrm{mg}, 96 \%$; $\mathrm{mp}>300{ }^{\circ} \mathrm{C}\left(\mathrm{MeOCH}_{2} \mathrm{CH}_{2} \mathrm{OH}\right) ;{ }^{1} \mathrm{H}-\mathrm{NMR}\left(300 \mathrm{MHz}\right.$, DMSO-d $\left.d_{6}\right): \delta 3.73$ $(3 \mathrm{H}, \mathrm{s}, \mathrm{OMe}), 4.56\left(2 \mathrm{H}, \mathrm{d}, J=6.0 \mathrm{~Hz}, \mathrm{CH}_{2}\right), 6.90\left(2 \mathrm{H}, \mathrm{d}, J=8.7 \mathrm{~Hz}, \mathrm{H}-3^{\prime \prime}\right.$ and $\left.\mathrm{H}-5^{\prime \prime}\right), 7.35(2 \mathrm{H}, \mathrm{d}$, $J=8.7 \mathrm{~Hz}, \mathrm{H}-2^{\prime \prime}$ and $\left.\mathrm{H}-6^{\prime \prime}\right), 7.48-7.58\left(3 \mathrm{H}, \mathrm{m}, \mathrm{H}-3^{\prime}, \mathrm{H}-4^{\prime}\right.$ and $\left.\mathrm{H}-5^{\prime}\right), 8.03-8.12\left(2 \mathrm{H}, \mathrm{m}, \mathrm{H}-2^{\prime}\right.$ and $\left.\mathrm{H}-6^{\prime}\right)$, $9.25(1 \mathrm{H}, \mathrm{t}, J=6.0 \mathrm{~Hz}, \mathrm{NH}), 12.25(1 \mathrm{H}, \mathrm{s}, \mathrm{N}(4) \mathrm{H}) .{ }^{13} \mathrm{C}-\mathrm{NMR}\left(75 \mathrm{MHz}, \mathrm{DMSO}-d_{6}\right): \delta 42.9,55.0,113.6(2 \mathrm{C})$, 126.5 (2C), 128.8 (2C), 128.9 (2C), 129.5, 129.9, 130.4, 148.0, 152.9, 153.5 (brs), 158.4, 161.4. Combustion elemental analysis calculated for $\mathrm{C}_{18} \mathrm{H}_{16} \mathrm{~N}_{6} \mathrm{O}_{2}$ : $\mathrm{C}, 62.06 ; \mathrm{H}, 4.63 ; \mathrm{N}, 24.12$. Found: $\mathrm{C}, 61.96 ; \mathrm{H}, 4.88$; N, 23.93.

2-Phenyl-7-[(pyridin-4-ylmethyl)amino]-1,2,4-triazolo[1,5-a][1,3,5]triazin-5-one (61). Colorless crystalline powder; yield $556 \mathrm{mg}, 87 \%$; mp 293-294 ${ }^{\circ} \mathrm{C}(\mathrm{EtOH}) ;{ }^{1} \mathrm{H}-\mathrm{NMR}\left(300 \mathrm{MHz}, \mathrm{DMSO}-d_{6}\right): \delta 4.66(2 \mathrm{H}, \mathrm{d}$, $\left.J=5.7 \mathrm{~Hz}, \mathrm{CH}_{2}\right), 7.40\left(2 \mathrm{H}, \mathrm{d}, J=4.9 \mathrm{~Hz}, \mathrm{H}-3^{\prime \prime}\right.$ and $\left.\mathrm{H}-5^{\prime \prime}\right), 7.48-7.61\left(3 \mathrm{H}, \mathrm{m}, \mathrm{H}-3^{\prime}, \mathrm{H}-4^{\prime}\right.$ and $\left.\mathrm{H}-5^{\prime}\right)$, 8.02-8.15 (2H, m, H-2' and $\left.\mathrm{H}-6^{\prime}\right), 8.52\left(2 \mathrm{H}, \mathrm{d}, J=4.9 \mathrm{~Hz}, \mathrm{H}-2^{\prime \prime}\right.$ and $\left.\mathrm{H}-6^{\prime \prime}\right), 9.36(1 \mathrm{H}, \mathrm{t}, J=5.7 \mathrm{~Hz}, \mathrm{NH})$, $12.29(1 \mathrm{H}, \mathrm{s}, \mathrm{N}(4) \mathrm{H}) .{ }^{13} \mathrm{C}-\mathrm{NMR}\left(75 \mathrm{MHz}, \mathrm{DMSO}-d_{6}\right): \delta 42.5,122.1(2 \mathrm{C}), 126.5(2 \mathrm{C}), 128.8(2 \mathrm{C}), 129.5$, 130.5, 146.9, 148.4, 149.4 (2C), 153.0, 153.4 (2C), 161.5. Combustion elemental analysis calculated for $\mathrm{C}_{16} \mathrm{H}_{13} \mathrm{~N}_{7} \mathrm{O}: \mathrm{C}, 60.18 ; \mathrm{H}, 4.10 ; \mathrm{N}, 30.70$. Found: C, 59.97; H, 4.34; N, 30.51.

2-Phenyl-7-phenylamino-1,2,4-triazolo[1,5-a][1,3,5]triazin-5-one (6m). Colorless crystalline powder; yield $545 \mathrm{mg}, 90 \%$; mp 298-299 ${ }^{\circ} \mathrm{C}\left(\mathrm{MeOCH}_{2} \mathrm{CH}_{2} \mathrm{OH}\right) ;{ }^{1} \mathrm{H}-\mathrm{NMR}\left(300 \mathrm{MHz}, \mathrm{DMSO}-d_{6}\right): \delta 7.23(1 \mathrm{H}, \mathrm{t}$, $\left.J=7.9 \mathrm{~Hz}, \mathrm{H}-4^{\prime \prime}\right), 7.43\left(2 \mathrm{H}, \mathrm{t}, J=7.9 \mathrm{~Hz}, \mathrm{H}-3^{\prime \prime}\right.$ and $\left.\mathrm{H}-5^{\prime \prime}\right), 7.49-7.61\left(3 \mathrm{H}, \mathrm{m}, \mathrm{H}-3^{\prime}, \mathrm{H}-4^{\prime}\right.$ and $\left.\mathrm{H}-5^{\prime}\right), 7.80$ $\left(2 \mathrm{H}, \mathrm{d}, J=7.9 \mathrm{~Hz}, \mathrm{H}-2^{\prime \prime}\right.$ and $\left.\mathrm{H}-4^{\prime \prime}\right), 8.09-8.21\left(2 \mathrm{H}, \mathrm{m}, \mathrm{H}-2^{\prime}\right.$ and $\left.\mathrm{H}-6^{\prime}\right), 10.44(1 \mathrm{H}, \mathrm{s}, \mathrm{NH}), 12.45(1 \mathrm{H}, \mathrm{s}$, $\mathrm{N}(4) \mathrm{H}) .{ }^{13} \mathrm{C}-\mathrm{NMR}\left(75 \mathrm{MHz}, \mathrm{DMSO}-d_{6}\right): \delta 123.3(2 \mathrm{C}), 125.2,126.7(2 \mathrm{C}), 128.4(2 \mathrm{C}), 128.8(2 \mathrm{C}), 129.5$, 130.5, 136.4, 146.5, 153.1, 153.3 (2C), 161.4. Combustion elemental analysis calculated for $\mathrm{C}_{16} \mathrm{H}_{12} \mathrm{~N}_{6} \mathrm{O}$ : C, 63.15; H, 3.97; N, 27.62. Found: C, 62.99; H, 4.24; N, 27.41.

7-(4-Chlorophenylamino)-2-phenyl-1,2,4-triazolo[1,5-a][1,3,5]triazin-5-one (6n). Colorless crystalline powder; yield $562 \mathrm{mg}$, 83\%; $\mathrm{mp}>300{ }^{\circ} \mathrm{C}\left(\mathrm{MeOCH}_{2} \mathrm{CH}_{2} \mathrm{OH}\right) ;{ }^{1} \mathrm{H}-\mathrm{NMR}\left(300 \mathrm{MHz}\right.$, DMSO- $\left.d_{6}\right)$ : $\delta 7.48\left(2 \mathrm{H}, \mathrm{d}, J=8.7 \mathrm{~Hz}, \mathrm{H}-3^{\prime \prime}\right.$ and $\left.\mathrm{H}-5^{\prime \prime}\right), 7.52-7.61\left(3 \mathrm{H}, \mathrm{m}, \mathrm{H}-3^{\prime}, \mathrm{H}-4^{\prime}\right.$ and $\left.\mathrm{H}-5^{\prime}\right), 7.87(2 \mathrm{H}, \mathrm{d}, J=8.7 \mathrm{~Hz}$, $\mathrm{H}-2^{\prime \prime}$ and H-4" $), 8.08-8.20\left(2 \mathrm{H}, \mathrm{m}, \mathrm{H}-2^{\prime}\right.$ and $\left.\mathrm{H}-6^{\prime}\right), 10.55(1 \mathrm{H}, \mathrm{s}, \mathrm{NH}), 12.49(1 \mathrm{H}, \mathrm{s}, \mathrm{N}(4) \mathrm{H}) .{ }^{13} \mathrm{C}-\mathrm{NMR}$ (75 MHz, DMSO- $d_{6}$ ): $\delta 124.7$ (2C), 126.7 (2C) 128.4 (2C), 128.8 (2C), 129.0, 129.5, 130.6, 135.5, 146.4, 153.1 (2C), 161.5. Combustion elemental analysis calculated for $\mathrm{C}_{16} \mathrm{H}_{11} \mathrm{ClN}_{6} \mathrm{O}: \mathrm{C}, 56.73 ; \mathrm{H}, 3.27 ; \mathrm{N}, 24.81$. Found: $\mathrm{C}, 56.62 ; \mathrm{H}, 3.36$; $\mathrm{N}, 24.70$.

7-(4-Methoxyphenylamino)-2-phenyl-1,2,4-triazolo[1,5-a][1,3,5]triazin-5-one (6o). Colorless crystalline powder; yield $650 \mathrm{mg}$, 96\%; mp 296-297 ${ }^{\circ} \mathrm{C}\left(\mathrm{MeOCH}_{2} \mathrm{CH}_{2} \mathrm{OH}\right) ;{ }^{1} \mathrm{H}-\mathrm{NMR}\left(300 \mathrm{MHz}\right.$, DMSO- $\left.d_{6}\right): \delta$ $3.78(3 \mathrm{H}, \mathrm{s}, \mathrm{OMe}), 6.99\left(2 \mathrm{H}, \mathrm{d}, J=8.7 \mathrm{~Hz}, \mathrm{H}-3^{\prime \prime}\right.$ and $\left.\mathrm{H}-5^{\prime \prime}\right), 7.49-7.60\left(3 \mathrm{H}, \mathrm{m}, \mathrm{H}-3^{\prime}, \mathrm{H}-4^{\prime}\right.$ and $\left.\mathrm{H}-5^{\prime}\right), 7.65$ $\left(2 \mathrm{H}, \mathrm{d}, J=8.7 \mathrm{~Hz}, \mathrm{H}-2^{\prime \prime}\right.$ and $\left.\mathrm{H}-4^{\prime \prime}\right), 8.08-8.19\left(2 \mathrm{H}, \mathrm{m}, \mathrm{H}-2^{\prime}\right.$ and $\left.\mathrm{H}-6^{\prime}\right), 10.35(1 \mathrm{H}, \mathrm{s}, \mathrm{NH}), 12.37(1 \mathrm{H}, \mathrm{s}$, $\mathrm{N}(4) \mathrm{H}) .{ }^{13} \mathrm{C}-\mathrm{NMR}\left(75 \mathrm{MHz}, \mathrm{DMSO}-d_{6}\right): \delta 55.2,113.6(2 \mathrm{C}), 125.0(2 \mathrm{C}), 126.7(2 \mathrm{C}), 128.8(2 \mathrm{C}), 129.1,129.5$, 130.6, 146.6, 153.1, $153.3(2 \mathrm{C}), 156.8,161.4$. Combustion elemental analysis calculated for $\mathrm{C}_{17} \mathrm{H}_{14} \mathrm{~N}_{6} \mathrm{O}_{2}$ : C, 61.07; H, 4.22; N, 25.14. Found: C, 60.87; H, 4.49; N, 24.98.

7-Indolino-2-phenyl-1,2,4-triazolo[1,5-a][1,3,5]triazin-5-one (6p). Colorless crystalline powder; yield $588 \mathrm{mg}, 89 \%$; mp $297^{\circ} \mathrm{C}\left(\mathrm{MeOCH}_{2} \mathrm{CH}_{2} \mathrm{OH}\right) ;{ }^{1} \mathrm{H}-\mathrm{NMR}\left(300 \mathrm{MHz}, \mathrm{DMSO}-d_{6}\right): \delta 3.27(2 \mathrm{H}, \mathrm{t}, J=8.3 \mathrm{~Hz}$, 
$\left.\mathrm{C}\left(3^{\prime \prime}\right) \mathrm{H}_{2}\right), 4.91\left(2 \mathrm{H}, \mathrm{d}, J=8.3 \mathrm{~Hz}, \mathrm{C}\left(2^{\prime \prime}\right) \mathrm{H}_{2}\right), 7.13\left(1 \mathrm{H}, \mathrm{t}, J=7.3 \mathrm{~Hz}, \mathrm{H}-5^{\prime \prime}\right), 7.27\left(1 \mathrm{H}, \mathrm{t}, J=7.7 \mathrm{~Hz}, \mathrm{H}-6^{\prime \prime}\right)$, $7.34\left(1 \mathrm{H}, \mathrm{d}, \mathrm{J}=7.2 \mathrm{~Hz}, \mathrm{H}-5^{\prime \prime}\right), 7.44-7.60\left(3 \mathrm{H}, \mathrm{m}, \mathrm{H}-3^{\prime}, \mathrm{H}-4^{\prime}\right.$ and $\left.\mathrm{H}-5^{\prime}\right), 8.00-8.15\left(2 \mathrm{H}, \mathrm{m}, \mathrm{H}-2^{\prime}\right.$ and H-6'), $8.40\left(1 \mathrm{H}, \mathrm{d}, J=7.9 \mathrm{~Hz}, \mathrm{H}-7^{\prime \prime}\right), 12.48(1 \mathrm{H}, \mathrm{s}, \mathrm{N}(4) \mathrm{H}) .{ }^{13} \mathrm{C}-\mathrm{NMR}\left(75 \mathrm{MHz}, \mathrm{DMSO}-d_{6}\right): \delta 27.9,52.3$, 118.9, 124.7, 124.8, 126.5 (2C), 126.7, 128.8 (2C), 129.3, 130.5, 133.1, 141.7, 146.1, 153.0, 154.6 (2C), 160.5. Combustion elemental analysis calculated for $\mathrm{C}_{18} \mathrm{H}_{14} \mathrm{~N}_{6} \mathrm{O}: \mathrm{C}, 65.44 ; \mathrm{H}, 4.27 ; \mathrm{N}, 25.44$. Found: $\mathrm{C}, 65.23$; $\mathrm{H}, 4.54 ; \mathrm{N}, 25.19$.

Author Contributions: Conceptualization, A.V.D.; Methodology, F.P.L.L. and A.V.D.; Investigation, A.J., Y.P.Z. and A.V.D.; Resources, W.K.C. and A.V.D.; Data Curation, F.P.L.L., Y.P.Z. and A.V.D.; Writing-Original Draft Preparation, A.J. and A.V.D.; Writing-Review and Editing, F.P.L.L., and A.V.D.; Supervision, W.K.C. and A.V.D.; Project Administration, W.K.C. and A.V.D.

Funding: This research was funded by the Ministry of Higher Education, Malaysia under Fundamental Research Grant Scheme, grant number FRGS/1/2015/SG01/MUSM/03/1.

Conflicts of Interest: The authors declare no conflict of interest.

\section{References}

1. Lim, F.P.L.; Dolzhenko, A.V. 1,3,5-Triazine-based analogues of purine: From isosteres to privileged scaffolds in medicinal chemistry. Eur. J. Med. Chem. 2014, 85, 371-390. [CrossRef] [PubMed]

2. Dolzhenko, A.V.; Dolzhenko, A.V.; Chui, W.K. Pyrazolo[1,5-a][1,3,5]triazines (5-aza-9-deazapurines). Synthesis and biological activity. Heterocycles 2008, 75, 1575-1622.

3. Dolzhenko, A.V.; Dolzhenko, A.V.; Chui, W.K. 1,2,4-Triazolo[1,5- $a][1,3,5]$ triazines (5-azapurines): Synthesis and biological activity. Heterocycles 2006, 68, 1723-1753. [CrossRef]

4. Kow, K.K.; Dolzhenko, A.V. Advances in the synthesis of 1,2,4-triazolo[1,5-a][1,3,5]triazines (5-azapurines) and their biological activity. Heterocycles 2019, 98, 175-203.

5. Lim, F.P.L.; Tan, K.C.; Luna, G.; Tiekink, E.R.T.; Dolzhenko, A.V. A synthesis of new 7-amino-substituted 4-aminopyrazolo[1,5-a][1,3,5]triazines via a selective three-component triazine ring annulation. Tetrahedron 2019, 75, 2322-2329. [CrossRef]

6. Lim, F.P.L.; Halcovitch, N.R.; Tiekink, E.R.T.; Dolzhenko, A.V. A new microwave-assisted, three-component reaction of 5-aminopyrazole-4-carboxylates: Selective synthesis of substituted 5-aza-9-deaza-adenines. Tetrahedron 2018, 74, 1868-1879. [CrossRef]

7. Lim, F.P.L.; Luna, G.; Dolzhenko, A.V. A one-pot, three-component, microwave-assisted synthesis of novel 7-amino-substituted 4-aminopyrazolo[1,5-a][1,3,5]triazine-8-carbonitriles. Tetrahedron Lett. 2015, 56, 7016-7019. [CrossRef]

8. Lim, F.P.L.; Luna, G.; Dolzhenko, A.V. A one-pot, three-component aminotriazine annulation onto 5-aminopyrazole-4-carbonitriles under microwave irradiation. Tetrahedron Lett. 2015, 56, 521-524. [CrossRef]

9. Lim, F.P.L.; Luna, G.; Dolzhenko, A.V. A new, one-pot, multicomponent synthesis of 5-aza-9-deaza-adenines under microwave irradiation. Tetrahedron Lett. 2014, 55, 5159-5163. [CrossRef]

10. Kalinina, S.A.; Kalinin, D.V.; Dolzhenko, A.V. A one-pot, three-component, microwave-promoted synthesis of 2-amino-substituted 7-amino-1,2,4-triazolo[1,5-a][1,3,5]triazines. Tetrahedron Lett. 2013, 54, 5537-5540. [CrossRef]

11. Dolzhenko, A.V.; Kalinina, S.A.; Kalinin, D.V. A novel multicomponent microwave-assisted synthesis of 5-aza-adenines. Rsc Adv. 2013, 3, 15850-15855. [CrossRef]

12. Bera, H.; Dolzhenko, A.V.; Sun, L.; Gupta, S.D.; Chui, W.K. Synthesis and in vitro evaluation of 1,2,4-triazolo[1,5-a][1,3,5]triazine derivatives as thymidine phosphorylase inhibitors. Chem. Biol. Drug Des. 2013, 82, 351-360. [CrossRef]

13. Bera, H.; Chui, W.K.; Gupta, S.D.; Dolzhenko, A.V.; Sun, L. Synthesis, in vitro evaluation of thymidine phosphorylase inhibitory activity, and in silico study of 1,3,5-triazin-2,4-dione and its fused analogues. Med. Chem. Res. 2013, 22, 6010-6021. [CrossRef]

14. Bera, H.; Tan, B.J.; Sun, L.; Dolzhenko, A.V.; Chui, W.K.; Chiu, G.N.C. A structure-activity relationship study of 1,2,4-triazolo[1,5-a][1,3,5]triazin-5,7-dione and its 5-thioxo analogues on anti-thymidine phosphorylase and associated anti-angiogenic activities. Eur. J. Med. Chem. 2013, 67, 325-334. [CrossRef] [PubMed] 
15. Kalinin, D.V.; Kalinina, S.A.; Dolzhenko, A.V. A new synthesis of amino substituted azolo[1,3,5]triazines via reaction of N1,N1-dimethyl-N2-azolylformamidines with cyanamide. Heterocycles 2013, 87, 147-154.

16. Zavodskaya, A.V.; Bakharev, V.V.; Parfenov, V.E.; Gidaspov, A.A.; Slepukhin, P.A.; Isenov, M.L.; Eltsov, O.S. Synthesis of new 5-aza-isosteres of guanine containing aryl and hetaryl substituents on the 1,2,4-triazole ring. Tetrahedron Lett. 2015, 56, 1103-1106. [CrossRef]

17. Bakharev, V.V.; Parfenov, V.E.; Ul'yankina, I.V.; Zavodskaya, A.V.; Selezneva, E.V.; Gidaspov, A.A.; Eltsov, O.S.; Slepukhin, P.A. Synthesis of new 5-aza-isosteres of guanine-5-aminosubstituted 1,2,4-triazolo[1,5-a]-1,3,5-triazin-7-ones. Tetrahedron 2014, 70, 6825-6830. [CrossRef]

18. Harauchi, Y.; Kajimoto, T.; Ohta, E.; Kawachi, H.; Imamura-Jinda, A.; Ohta, S. Prenylated purine alkaloids from seeds of Gleditsia japonica. Phytochemistry 2017, 143, 145-150. [CrossRef] [PubMed]

19. Kajimoto, T.; Aoki, N.; Ohta, E.; Ohta, S. Locustoside A-A new purine alkaloid glucoside from seeds of Gleditsia japonica. Phytochem. Lett. 2010, 3, 198-200. [CrossRef]

20. Kajimoto, T.; Aoki, N.; Ohta, E.; Kawai, Y.; Ohta, S. Saikachinoside A, a novel 3-prenylated isoguanine glucoside from seeds of Gleditsia japonica. Tetrahedron Lett. 2010, 51, 2099-2101. [CrossRef]

21. Pereira, H.M.; Berdini, V.; Cleasby, A.; Garratt, R.C. Crystal structure of calf spleen purine nucleoside phosphorylase complexed to a novel purine analogue. FEBS Lett. 2007, 581, 5082-5086. [CrossRef]

22. Furman, R.R.; Hoelzer, D. Purine nucleoside phosphorylase inhibition as a novel therapeutic approach for B-cell lymphoid malignancies. Semin. Oncol. 2007, 34, S29-S34. [CrossRef]

23. Roberts, E.L.L.; Newton, R.P.; Axford, A.T. Plasma purine nucleoside phosphorylase in cancer patients. Clin. Chim. Acta 2004, 344, 109-114. [CrossRef]

24. Bzowska, A. Formycins and their analogues: Purine nucleoside phosphorylase inhibitors and their potential application in immunosuppression and cancer. In Modified Nucleosides: In Biochemistry, Biotechnology and Medicine; Herdewijn, P., Ed.; Wiley-VCH Verlag GmbH \& Co. KGaA: Weinheim, Germany, 2008; pp. 473-510.

25. Donaldson, T.M.; Cassera, M.B.; Ho, M.-C.; Zhan, C.; Merino, E.F.; Evans, G.B.; Tyler, P.C.; Almo, S.C.; Schramm, V.L.; Kim, K. Inhibition and structure of Toxoplasma gondii purine nucleoside phosphorylase. Eukaryot. Cell 2014, 13, 572-579. [CrossRef]

26. Lim, F.P.L.; Dolzhenko, A.V. 4-Amino-substituted pyrazolo[1,5- $a][1,3,5]$ triazin-2-amines: A new practical synthesis and biological activity. Tetrahedron Lett. 2014, 55, 6684-6688. [CrossRef]

27. Dolzhenko, A.V.; Pastorin, G.; Dolzhenko, A.V.; Chui, W.K. A convenient method for the synthesis of 7-amino-substituted 1,2,4-triazolo[1,5-a][1,3,5]triazin-5-amines. Tetrahedron Lett. 2008, 49, 7180-7183. [CrossRef]

28. Dolzhenko, A.V.; Dolzhenko, A.V.; Chui, W.K. Practical synthesis of regioisomeric 5(7)-amino-6,7(4,5)-dihydro[1,2,4]triazolo[1,5-a][1,3,5]triazines. Tetrahedron 2007, 63, 12888-12895. [CrossRef]

29. Dolzhenko, A.V.; Dolzhenko, A.V.; Chui, W.K. Synthesis of 5,7-diamino[1,2,4]triazolo[1,2-a]-[1,3,5]triazines via annulation of 1,3,5-triazine ring onto 3(5)-amino-1,2,4-triazoles. Heterocycles 2007, 71, 429-436. [CrossRef]

30. Caulkett, P.W.R.; Jones, G.; McPartlin, M.; Renshaw, N.D.; Stewart, S.K.; Wright, B. Adenine isosteres with bridgehead nitrogen. Part 1 . Two independent syntheses of the $[1,2,4]$ traizolo[1,5- $a][1,3,5]$ triazine ring system leading to a range of substituents in the 2, 5 and 7 positions. J. Chem. Soc. Perkin Trans. 1 Org. Bio-Org. Chem. 1995, 1, 801-808.

31. Federico, S.; Ciancetta, A.; Porta, N.; Redenti, S.; Pastorin, G.; Cacciari, B.; Klotz, K.N.; Moro, S.; Spalluto, G. 5,7-Disubstituted-[1,2,4]triazolo[1,5- $a][1,3,5]$ triazines as pharmacological tools to explore the antagonist selectivity profiles toward adenosine receptors. Eur. J. Med. Chem. 2016, 108, 529-541. [CrossRef]

32. Redenti, S.; Marcovich, I.; De Vita, T.; Pérez, C.; De Zorzi, R.; Demitri, N.; Perez, D.I.; Bottegoni, G.; Bisignano, P.; Bissaro, M.; et al. A triazolotriazine-based dual GSK-3 $\beta / C K-1 \delta$ ligand as a potential neuroprotective agent presenting two different mechanisms of enzymatic inhibition. ChemMedChem 2019, 14, 310-314. [CrossRef]

33. Lefoix, M.; Mathis, G.; Kleinmann, T.; Truffert, J.-C.; Asseline, U. Pyrazolo[1,5-a]-1,3,5-triazine C-nucleoside as deoxyadenosine analog: Synthesis, pairing, and resistance to hydrolysis. J. Org. Chem. 2014, 79, 3221-3227. [CrossRef]

34. Popowycz, F.; Schneider, C.; De Bonis, S.; Skoufias, D.A.; Kozielski, F.; Galmarini, C.M.; Joseph, B. Synthesis and antiproliferative evaluation of pyrazolo[1,5-a]-1,3,5-triazine myoseverin derivatives. Bioorg. Med. Chem. 2009, 17, 3471-3478. [CrossRef] 
35. Popowycz, F.; Fournet, G.; Schneider, C.; Bettayeb, K.; Ferandin, Y.; Lamigeon, C.; Tirado, O.M.; Mateo-Lozano, S.; Notario, V.; Colas, P.; et al. Pyrazolo[1,5-a]-1,3,5-triazine as a purine bioisostere: Access to potent cyclin-dependent kinase inhibitor (R)-roscovitine analogue. J. Med. Chem. 2009, 52, 655-663. [CrossRef]

36. Raboisson, P.; Schultz, D.; Muller, C.; Reimund, J.-M.; Pinna, G.; Mathieu, R.; Bernard, P.; Do, Q.-T.; DesJarlais, R.L.; Justiano, H.; et al. Cyclic nucleotide phosphodiesterase type 4 inhibitors: Evaluation of pyrazolo[1,5-a]-1,3,5-triazine ring system as an adenine bioisostere. Eur. J. Med. Chem. 2008, 43, 816-829. [CrossRef]

37. Mathieu, R.; Schmitt, M.; Bourguignon, J.-J. Stereoselective synthesis of 3'-substituted 2'-deoxy C-nucleoside pyrazolo[1,5-a]-1,3,5-triazines and their 5'-phosphate nucleotides. Tetrahedron Lett. 2006, 47, 5099-5103. [CrossRef]

38. Raboisson, P.; Schultz, D.; Lugnier, C.; Mathieu, R.; Bourguignon, J.-J. A general approach toward the synthesis of 8-amidopyrazolo[1,5-a]-1,3,5-triazines. Tetrahedron Lett. 2003, 44, 703-705. [CrossRef]

39. Raboisson, P.; Schultz, D.; Lugnier, C.; Bourguignon, J.-J. Efficient synthesis of 8-substituted pyrazolo[1,5-a]-1,3,5-triazines by regioselective acylation. Tetrahedron Lett. 2002, 43, 9501-9503. [CrossRef]

40. Raboisson, P.; Baurand, A.; Cazenave, J.-P.; Gachet, C.; Schultz, D.; Spiess, B.; Bourguignon, J.-J. A General approach toward the synthesis of C-nucleoside pyrazolo[1,5-a]-1,3,5-triazines and their 3',5'-bisphosphate C-nucleotide analogues as the first reported in vivo stable P2Y1-receptor antagonists. J. Org. Chem. 2002, 67, 8063-8071. [CrossRef]

41. Dolzhenko, A.V.; Tan, G.K.; Koh, L.L.; Chui, W.K. N-Carbethoxy-N'-(3-phenyl-1H-1,2,4-triazol-5-yl)thiourea. Acta Crystallogr. Sect. E 2010, 66, o425. [CrossRef]

42. Dolzhenko, A.V.; Tan, G.K.; Koh, L.L.; Chui, W.K. 2-Phenyl-7-(4-pyridylmethylamino)-1,2,4-triazolo [1,5-a][1,3,5]triazin-5(4H)-one. Acta Crystallogr. Sect. E 2011, 67, o85-086. [CrossRef]

Sample Availability: Samples of compounds $\mathbf{6 a}-\mathbf{p}$ are available from the authors.

(C) 2019 by the authors. Licensee MDPI, Basel, Switzerland. This article is an open access article distributed under the terms and conditions of the Creative Commons Attribution (CC BY) license (http://creativecommons.org/licenses/by/4.0/). 